\title{
Automatic 3D Registration of Lung Surfaces in Computed Tomography Scans
}

\author{
Margrit Betke, $\mathrm{PhD}^{1}$, Harrison Hong, $\mathrm{BA}^{1}$, and Jane P. Ko, $\mathrm{MD}^{2}$ \\ 1 Computer Science Department \\ Boston University, \\ Boston, MA 02215, USA \\ betke@cs.bu.edu \\ http://www.cs.bu.edu/faculty/betke \\ 2 Department of Radiology \\ New York University Medical School \\ New York, NY 10016, USA
}

\begin{abstract}
We developed an automated system that registers chest CT images temporally. Our registration method matches corresponding anatomical landmarks to obtain initial registration parameters. The initial point-to-point registration is then generalized to an iterative surface-tosurface registration method. Our "goodness-of-fit" measure is evaluated at each step in the iterative scheme until the registration performance is sufficient. We applied our method to register the 3D lung surfaces of 10 pairs of chest CT scans and report a promising registration performance 1
\end{abstract}

\section{Introduction}

Chest computed tomography (CT) has become a well-established means of diagnosing pulmonary metastasis of oncology patients and evaluating response to treatment regimens. Since diagnosis and prognosis of cancer generally depend upon growth assessment, repeated CT studies are used to determine growth rates of pulmonary nodules. Chest $\mathrm{CT}$ is currently being evaluated as a method for screening for lung cancer. Lung cancer remains the leading cause of cancer death in the United States, killing 160,000 people a year. The overall 5-year survival rate is $15 \%$, but early detection and resection can improve the prognosis significantly. For example, the 5-year survival rate for Stage I cancer is $67 \%$ 13.

Our long-term objective is to develop an image analysis system that assists the radiologist in detecting and comparing pulmonary nodules in repeated CT studies in a clinical setting. Such a system must solve the classical problems in medical image analysis - segmentation, detection, and registration - for the important domain of chest CT images. References [4] and [10] describe our preliminary system. It automatically segments the thorax, lungs, and structures

\footnotetext{
${ }^{1}$ The support of the Office of Naval Research, National Science Foundation, Radiological Society of North America, and the Whitaker Foundation is gratefully acknowledged.
} 
within the lungs, and detects nodules in axial chest CT images. Human intervention is needed to match up the studies. In the current paper, we focus on automating the registration task. In particular, we describe a method for automatic three-dimensional (3D) alignment of lung surfaces in repeated CT scans.

A large body of literature has been published on registration techniques [1, 2, 3, 6, 7, 8, 9, 11, 14, 15. Here we can only point to some approaches that are most closely related to our work, e.g., approaches that use anatomical landmarks for registration [7], register points to points [8] or surfaces [2, 11], and correlate subimages [1]. Often only a small misalignment of the images is assumed [1]. Other registration methods require some manual input to compensate for rotational and translational differences between two studies [14]. Earlier surveys on registration are [6, 15].

Medical registration techniques have primarily been developed for the brain. Registration of chest radiographs has been addressed by Kano et al. 9]. To the best of our knowledge, an automated system to register chest CT images temporally has not been developed yet. Registration of thoracic CT studies is challenging, since patient position varies each time a study is taken in terms of differences in torso rotation and translation. Differences in inspiratory volumes between two studies are other obstacles to registration.

In this work, the lung surfaces of two CT scans were segmented and registered for 10 patients. We first describe an automatic landmark-based registration method, then generalize it to surface-to-surface registration, and improve it with an iterative algorithm, which extends Besl's registration scheme [2]. Finally, we report and discuss the registration results for the 10 pairs of chest CT scans.

\section{Methods}

Registration of Anatomical Landmarks. Registration techniques determine the absolute orientation of one data set with respect to the other. The 3D coordinates of corresponding points in the two different data sets are known. For our $3 \mathrm{D}$ data sets, it is difficult to establish the anatomical correspondence of voxels, even for a human observer. We therefore use the voxels that make up anatomical landmarks for our initial registration method. We do not use external fiduciary markers, which would be impractical in a clinical setting.

Bones are rigid anatomical features that can be registered reliably. In particular, the sternum and vertebrae are excellent anatomical landmarks, because their positions are relatively fixed within the chest. We also use the trachea as an anatomical landmark. Although the position and shape of the trachea change with respiration, the trachea centroid serves as a reliable landmark for registration of our data sets. Finally, we also tested the use of structures within the lungs, for example nodules, for registration. Figure 1 shows how the centroids of sternum, trachea, and a nodule in the left lung are registered in corresponding axial images of two CT data sets. 


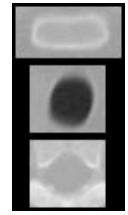

(a)

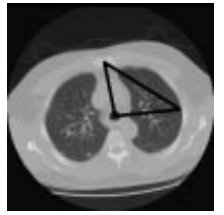

(b)

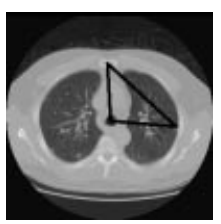

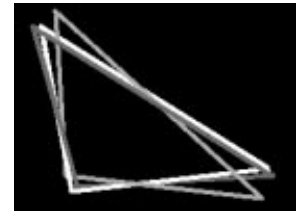

(c)

Fig. 1. (a) Template images of the sternum, trachea, and spine. (b) Corresponding CT images in two studies. The landmarks are the centroids of the sternum, trachea, and a nodule in the left lung. (c) The light grey test points in study 2 must be matched to the white model points in study 1 . The best transformation of the test points is shown in dark grey.

Correlation-Based Recognition of Anatomical Features. We use template images of anatomical landmarks, such as sternum, vertebra, and trachea shown in Fig. 1, to detect these landmarks in our test data. The template images are created offline by manually cropping subimages of the features out of a training data set. Although the features look slightly different in the test data, training and test data generally match well. This is particularly true if we use a deformable template that can be scaled or rotated.

Let a describe the affine parameters position, scale, and rotation of the template. We use the normalized correlation coefficient to find the best estimate of the affine parameters. In our previous work [5], we showed when the statistically optimal estimator for the affine parameters takes the form of the normalized correlation coefficient. It quantifies how well the measured data in subimage $I_{q}(x, y)$ matches the template feature in $q(x, y ; \mathbf{a})$. The normalized correlation coefficient is defined by $r(\mathbf{a})=1 /\left(\sigma_{I}(\mathbf{a}) \sigma_{q}(\mathbf{a})\left(A(\mathbf{a}) \sum_{(x, y) \in O} I_{q}(x, y) q(x, y ; \mathbf{a})-\right.\right.$ $\left.m_{I}(\mathbf{a}) m_{q}(\mathbf{a})\right)$, where $m_{I}(\mathbf{a})=\sum I_{q}(x, y)$ and $m_{q}(\mathbf{a})=\sum q(x, y ; \mathbf{a})$ are the respective local image means, $\sigma_{I}^{2}(\mathbf{a})=A(\mathbf{a}) \sum I_{q}(x, y)^{2}-\left(\sum I_{q}(x, y)\right)^{2}$ and $\sigma_{q}^{2}(\mathbf{a})=A(\mathbf{a}) \sum q(x, y ; \mathbf{a})^{2}-\left(\sum q(x, y ; \mathbf{a})\right)^{2}$ are the respective local variances, and where the sums are computed over a region $O$ that is the union of all pixels that contain the expected feature and $A=|O|$ is the number of pixels in $O$.

The ambiguity surfaces for the position estimates of anatomical features have global peaks with correlations of at least 0.8 , which lie far above the expected correlation $E[r(\mathbf{a})]=0$. In addition, once a feature, such as the trachea, is found in some axial image, the search space for the same feature in subsequent images can be reduced significantly. In addition, we update the template feature $q$ automatically online with the cropped image of the detected feature in the previous slice. This results in high correlations $(\geq 0.9)$ and reliable estimates of feature position and size.

Three-Dimensional Affine Point-to-Point Registration. Given a voxel $\mathbf{x}$ in study 1 and a voxel $\mathbf{p}$ in study 2 , the general $3 \mathrm{D}$ affine transformation $\mathbf{x}=$ $\mathbf{A p}+\mathbf{x}_{\mathbf{0}}$ maps $\mathbf{p}$ into $\mathbf{x}$, where the $3 \times 3$ matrix $\mathbf{A}$ can be expressed in terms 
of nine parameters, three for rotation, three for scaling, and three for skewing. Vector $\mathbf{x}_{\mathbf{0}}$ describes the $3 \mathrm{D}$ translation between $\mathbf{x}$ and $\mathbf{p}$.

In our application, the rotation parameters model the orientation of the patient's body on the CT table. Scaling in the $x$ and $y$ dimensions models changes in the field-of-view, i.e., the pixel-width-to-millimeter ratio. Scaling in $z$ is due to the differing slice thickness and number of slices in the two studies. Since the scaling parameters are determined before scan acquisition, we do not need to invert for the scaling parameters. We assume that the CT scanner does not introduce skewing and preserves the Cartesian coordinates of $3 \mathrm{D}$ points. Then the problem of finding the general affine transformation reduces to finding the rigid-body transformation after the 2 studies have been adjusted for scaling differences. The rigid-body transformation $\mathcal{T}$ maps $\mathbf{p}$ into $\mathbf{x}$,

$$
\mathbf{x}=\mathcal{T}(\mathbf{p})=\mathbf{R} \mathbf{p}+\mathbf{x}_{\mathbf{0}},
$$

where the orthonormal $3 \times 3$ matrix $\mathbf{R}$ rotates $\mathbf{p}$ into vector $\mathbf{R} \mathbf{p}$, which is then shifted into $\mathbf{x}$ by translation vector $\mathbf{x}_{\mathbf{0}}$. We have 12 unknowns (9 matrix coefficients and 3 translation parameters) and only 3 linear equations. So we need at least 4 corresponding points to compute the unknown transformation parameters. If we impose the orthonormality condition, we obtain an additional equation and therefore only need 3 corresponding points.

Since there may be errors in the measurement of the points or in the corresponding landmark detection algorithm, a greater accuracy in determining the transformation parameters can be obtained if more than three points are used. Given a set $X$ of $n$ points $\mathbf{x}_{1}, \ldots, \mathbf{x}_{n}$ in study 1 and a set $P$ of corresponding points $\mathbf{p}_{1}, \ldots, \mathbf{p}_{n}$ in study 2 , we minimize the sum of square residual errors

$$
\sum_{i=1}^{n}\left\|\mathbf{e}_{i}\right\|^{2}=\sum_{i=1}^{n}\left\|\mathbf{x}_{i}-\mathcal{T}\left(\mathbf{p}_{i}\right)\right\|^{2}=\sum_{i=1}^{n}\left\|\mathbf{x}_{i}-\mathbf{R} \mathbf{p}_{i}-\mathbf{x}_{\mathbf{0}}\right\|^{2}
$$

with respect to the unknowns $\mathbf{R}$ and $\mathbf{x}_{\mathbf{0}}$. A closed-form optimal solution to this least-squares problem was given by Horn [8]. The best translation vector $\hat{\mathbf{x}}_{\mathbf{0}}$ is the difference between the centroid $\overline{\mathbf{x}}=1 / n \sum_{i=1}^{n} \mathbf{x}_{i}$ of point set $X$ and the centroid $\overline{\mathbf{p}}=1 / n \sum_{i=1}^{n} \mathbf{p}_{i}$ of point set $P$ rotated by rotation $\mathbf{R}$ :

$$
\mathbf{x}_{\mathbf{0}}=\overline{\mathbf{x}}-\mathbf{R}(\overline{\mathbf{p}}) .
$$

Therefore, the translation can be computed easily once the rotation is found. To find the rotation, the coordinates of voxels in $X$ and $P$ are converted into coordinates of voxels in $X^{\prime}$ and $P^{\prime}$ of coordinate systems that are originated at the respective centroids, e.g., $\mathbf{x}_{\mathbf{i}}^{\prime}=\mathbf{x}_{\mathbf{i}}-\overline{\mathbf{x}}$ for all $\mathbf{x}_{\mathbf{i}} \in X$. This reduces the least-squares problem of Eq. 2 to a minimization of $\sum_{i=1}^{n}\left\|\mathbf{x}_{i}^{\prime}-\mathbf{R} \mathbf{p}_{i}^{\prime}\right\|^{2}=$ $\sum_{i=1}^{n}\left\|\mathbf{x}_{i}^{\prime}\right\|^{2}-2 \sum_{i=1}^{n} \mathbf{x}_{i}^{\prime T} \mathbf{R} \mathbf{p}_{i}^{\prime}+\sum_{i=1}^{n}\left\|\mathbf{p}_{i}^{\prime}\right\|^{2}$ with respect to rotation $\mathbf{R}$ only, or $\max _{\mathbf{R}} \sum_{i=1}^{n} \mathbf{x}_{i}^{\prime T} \mathbf{R} \mathbf{p}_{i}^{\prime}$. The solution of this maximization problem is given by a unit quaternion (see details in [8, 3]). In Fig. 1] the centroids of the sternum, trachea, and a nodule in study 1 , shown in white, are registered to the corresponding centroids in study 2 , shown in light grey. The registration results are shown in dark grey. 


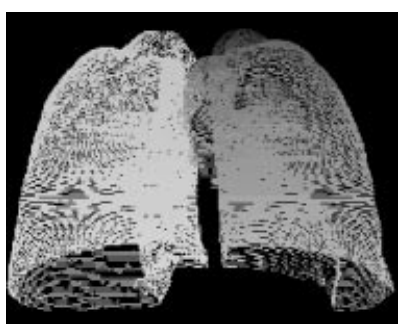

(a)

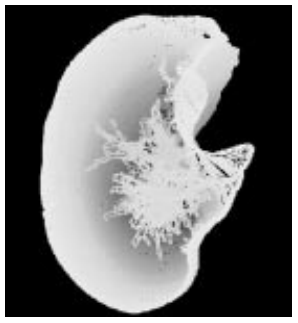

(b)

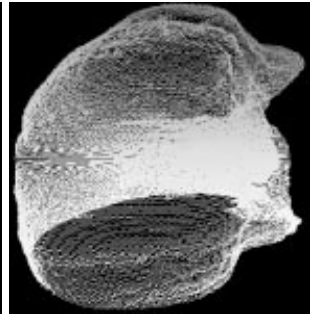

(c)

Fig. 2. 3D visualization of the lung. (a) Coronal view of both lungs, (b) topdown axial view of the right lung, and (c) wire-frame visualization of the right lung.

Three-Dimensional Affine Shape Registration. In this paper, we focus on lung border surfaces that are segmented from the full 3D data set using the method described in our ealier work [10, 4]. Figure 2 visualizes lung surfaces segmented from a low-dose CT scan. Our goal is to register such a surface to the lung surface of the same patient that is imaged at a later time.

The point-to-point registration algorithm described above assumes that the correspondence between points has been established. For certain points, e.g., the centroids of the sternum in corresponding axial slices, correspondence can be determined with relatively high confidence, but the correspondences of other point pairs are not as easily established. For example, a lung border point in the apex of study 1 corresponds to some border point in the apex of study 2, but which point generally cannot be determined, even by a human observer. We therefore define the correspondence $\mathcal{C}$ of two points on different surfaces by their distance. In particular, test point $\mathbf{p}_{i}$ corresponds to model point $\mathbf{x}_{j}=\mathcal{C}\left(\mathbf{p}_{i}\right)$, if their Euclidean distance is the shortest among all distances between $\mathbf{p}_{i}$ and any point in $X$, i.e., $\mathcal{C}\left(\mathbf{p}_{i}\right)=\mathbf{x}_{j}$, for which $\left\|\mathbf{x}_{j}-\mathbf{p}_{i}\right\|=\min _{\mathbf{x}_{k} \in X}\left\|\mathbf{x}_{k}-\mathbf{p}_{i}\right\|$. With this definition, we can match two surfaces that contain a different number of voxels.

The computed correspondences are reliable if the two data sets are close to each other, in particular, if they have been registered. This creates a paradoxical situation: we would like to register corresponding points, but need to register them first in order to establish their correspondences. To resolve this situation, we solve the registration and correspondence problems concurrently, an approach proposed by Besl [2]. We first detect anatomical landmarks in studies 1 and 2 and compute the 3D affine transformation that registers them optimally. We then segment the lungs [10, 4] and register them with the transformation parameters computed for the landmark registration. We establish correspondences by computing the Euclidean distances between all point pairs of the two data sets, register the transformed lung borders in study 2 to the lung borders in study 1, compute the new correspondences and error, and then iterate. Once the error is sufficiently small, the process is terminated. Note that this process does not guarantee that corresponding points are the same physical point. 

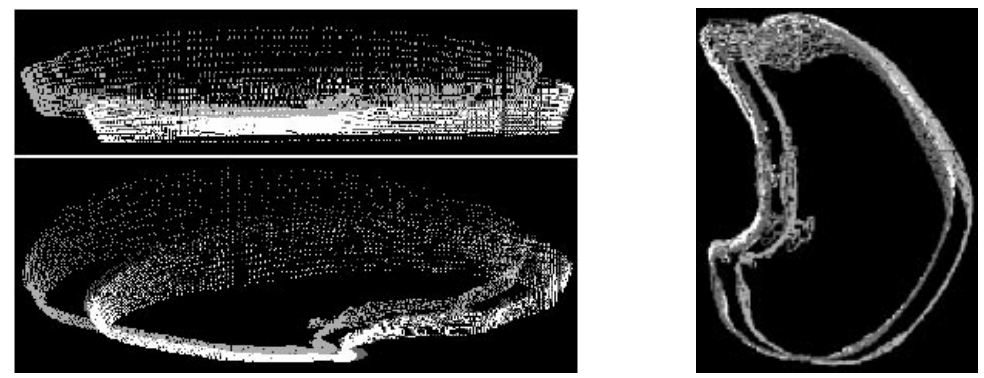

Fig. 3. Visualization of the $3 \mathrm{D}$ registration of 10 left lung border curves with views from the lung's side (top left), bottom (bottom left), and top (right). Points in study 2 (light grey) are registered to points in study 1 (white) by a transformation that maps them into points shown in dark grey. The registration error is minimal.

Function LungRegistration takes as inputs 3D voxel data sets CTstudy1 and CTstudy 1 that have been adjusted for field-of-view differences, and a parameter threshold that is used to decide when the function can terminate with a sufficient registration result. It outputs the transformation parameters for translation and rotation. For its function calls, we use C-style notation to distinguish input parameters, e.g., in line 5, lung1, from parameters that change during the function call, e.g., \&lungR, \&translation.

1 Function LungRegistration (CTstudy1, CTstudy2, threshold) \{

2 DetectLandmarks (\&landmarks1, \&landmarks2);

3 RegisterLandmarks(landmarks1, landmarks2, \&translation, \&rotation);

4 SegmentLungs(CTstudy1, CTstudy2, \&lung1, \&lung2);

5 RegisterLungsInitially(lung1, lung2, translation, rotation, \&lungR);

6 ComputeCorrespondencesAndError (lung1, lungR, \&error);

7 while (error $>$ threshold) \{

8 RegisterLungs (lung1, \&lungR, \&translation, \&rotation);

9 ComputeCorrespondencesAndError (lung1, lungR, \&error); \}

10 OutputResults(translation, rotation); \}

\section{Results}

Ten patients with cancer diagnoses and pulmonary nodules were selected, who had two thoracic CT scans for clinical indications between 1993 and 2001. A total of $20 \mathrm{CT}$ studies was evaluated. Fourteen chest CT scans had been performed helically on GE HiSpeed Advantage machines. The scans were obtained from the lung apices through the adrenal glands using a 1:1 pitch either with $5 \mathrm{~mm}$ collimation for the entire study or $10 \mathrm{~mm}$ collimation with $5 \mathrm{~mm}$ collimation through the hila. Six studies were taken on a multi-helical Siemens Somatom Volume Zoom CT and reconstructed in $1.0 \mathrm{~mm}$ intervals. The landmarks used for the 

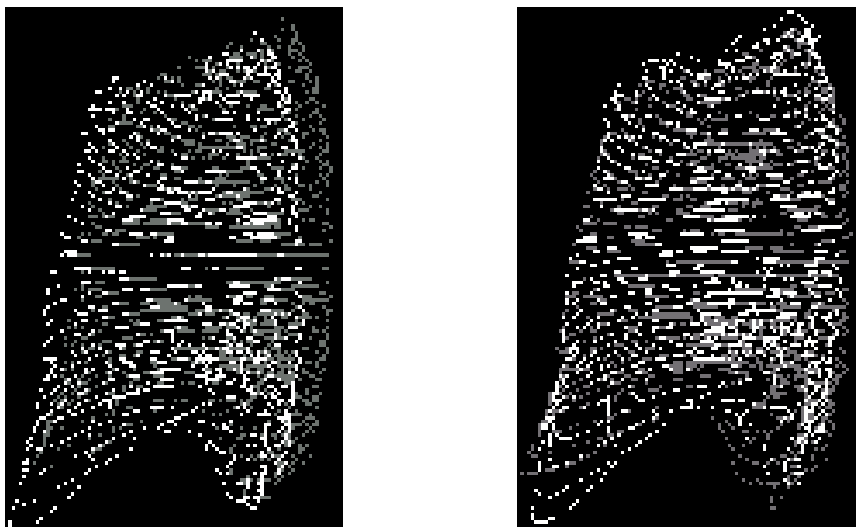

Fig. 4. On the left, wire models of the right lung for study 1 (grey) and study 2 (white). On the right, the transformed lung surface of study 2 (white). It matches well with the lung surface of study 1 (grey).

initial registration are the centroids of the trachea at the apex and just above the carina, and of the sternum and vertebra also in the slice above the carina. We evaluated the results quantitatively with the sum-squared-differences (SSD) measure (Eq. 2) and qualitatively by visual inspection. Table 1 summarizes the results. Using a fixed length of 25 iterations, the registration error was reduced up to $86 \%$ of the initial error. We also registered CT scans based on corresponding vessel landmarks that a radiologist identified and report the differences in registration results between human and computer. Visual inspection of the 3D lung registration results in Figs. 3 and 4 shows that the measured and computed points match well and confirms the results of our quantitative error analysis.

\section{Discussion and Conclusions}

To overcome the need of human intervention in our preliminary system [10, 4], we developed an automatic 3D registration method that matches the lung surfaces in repeated CT studies. Our results for 10 pairs of CT scans are very promising. In the future, we will also test if our system can reliably register corresponding nodules in repeated chest CT scans and thus become a clinically useful tool for nodule growth assessment.

We presented a global registration method - any change in a transformation parameter influences the transformation of the $3 \mathrm{D}$ data set as a whole 15 . In the future, we will design deformable surface models [12] to describe local transformations that are due to differences in patient respiration. We will then address the difficult task of registering structures within the lung. This will require modeling of nodule shape and position as a function of lung surface deformation, since nodules may move within the lung due to the patient's respiration. 
Table 1: Registration Results

\begin{tabular}{rccccrc}
\hline Patient & $\begin{array}{l}\text { Months } \\
\text { between } \\
\text { Studies }\end{array}$ & $\begin{array}{l}\text { Reconstruc- } \\
\text { tion interv. } \\
\text { in } m\end{array}$ & $\begin{array}{c}\text { Rotation } \\
\text { in Euler } \\
\text { Angles }\end{array}$ & $\begin{array}{c}\text { Trans- } \\
\text { lation } \\
\text { in } m m\end{array}$ & $\begin{array}{c}\text { SSD Error } \\
\text { Reduction } \\
\text { after 25 it. }\end{array}$ & $\begin{array}{c}\text { Transl. Error } \\
\text { Comp. vs. } \\
\text { Radiol. } m m\end{array}$ \\
\hline 1 & 2 & $10 / 5 / 10$ & $(2.3,0,9.3)$ & 15 & $21 \%$ & 10 \\
2 & $4 \frac{1}{2}$ & $10 / 5 / 10$ & $(-0.5,0.3,-2.4)$ & 26 & $34 \%$ & 8 \\
3 & $1 \frac{1}{2}$ & 5 & $(3.6,1.2,-8.6)$ & 6 & $86 \%$ & 9 \\
4 & $1 \frac{1}{2}$ & $10 / 5 / 10$ & $(0.2,-0.6,3.7)$ & 142 & $7 \%$ & - \\
5 & 4 & 1 & $(0.1,-4.0,1.9)$ & 5 & $20 \%$ & 2 \\
6 & 7 & 1 & $(-1.9,-1.4,1.8)$ & 3 & $51 \%$ & 3 \\
7 & $6 \frac{1}{2}$ & 1 & $(-6.7,-5.5,4.4)$ & 27 & $78 \%$ & 8 \\
8 & 2 & 5 & $(-1.8,3.6,-9.8)$ & 21 & $75 \%$ & 4 \\
9 & $1 \frac{1}{4}$ & 5 & $(0.2,0.1,4.5)$ & 58 & $8 \%$ & 6 \\
10 & 1 & 5 & $(5.2,1.5,3.3)$ & 14 & $81 \%$ & 4 \\
\hline
\end{tabular}

Landmark detection and registration significantly improve the speed of the registration process. Since there is a tradeoff between speed and precision of registration, we will test the impact of resolution reduction on registration performance. We will also investigate whether initial registration of a larger set of landmarks will improve registration precision and speed.

In summary, we have developed a 3D method for registration of lung surfaces in repeated chest $\mathrm{CT}$ scans and shown a promising registration performance for 10 patients.

\section{Acknowledgements}

The authors thank David Naidich, MD, Marilyn Noz, PhD, and Chekema Prince for their support.

\section{References}

[1] R. J. Althof, M. G. J. Wind, and J. T. Dobbins III. A rapid and automatic image registration algorithm with subpixel accuracy. IEEE Trans Med Imag, 16(3):308316, 1997.

[2] P. J. Besl and N. D. McKay. A method for registration of 3-D shapes. IEEE Trans Pattern Anal Mach Intell, 14(2):239-256, 1992.

[3] M. Betke, H. Hong, and J. P. Ko. Automatic 3D registration of lung surfaces in computed tomography scans. http://www.cs.bu.edu/techreports, 2001.

[4] M. Betke and J. P. Ko. Detection of pulmonary nodules on CT and volumetric assessment of change over time. In Medical Image Computing and ComputerAssisted Intervention - MICCAI'99, pages 245-252. Springer-Verlag, Berlin, 1999.

[5] M. Betke and N. C. Makris. Recognition, resolution and complexity of objects subject to affine transformation. Int J Comput Vis, 44(1), 2001.

[6] L. G. Brown. A survey of image registration techniques. ACM Computing Surveys, 24(4):325-375, December 1992. 
[7] W. R. Fright and A. D. Linney. Registration of 3-D head surfaces using multiple landmarks. IEEE Trans Med Imag, 12(3):515-520, 1993.

[8] B. K. P. Horn. Closed-form solution of absolute orientation using unit quaternions. J Opt Soc Am, 4(4):629-642, 1987.

[9] A. Kano, K. Doi, H. MacMahon, D. D. Hassell, and M. L. Giger. Digital image subtraction of temporally sequential chest imagers for detection of interval change. Med Phys, 21(3):453-461, 1994.

[10] J. P. Ko and M. Betke. Chest CT: Automated nodule detection and assessment of change over time - preliminary experience. Radiology, 218(1):267-273, 2001.

[11] C. R. Maurer, G. B. Aboutanos, B. M. Dawant, R. J. Maciunas, and J. M. Fitzpatrick. Registration of 3-D images using weighted geometrical features. IEEE Trans Med Imag, 15(6):836-849, 1996.

[12] D. N. Metaxas. Physics-Based Deformable Models: Applications to Computer Vision, Graphics, and Medical Imaging. Kluwer Academic Press, Boston, 1997.

[13] C. F. Mountain. Revisions in the international system for staging lung cancer. Chest, 111(6):1710-1717, June 1997.

[14] C. A. Pelizzari, G. T. Chen, D. R. Spelbring, R. R. Weichselbaum, and C. T. Chen. Accurate three-dimensional registration of CT, PET and/or MR images of the brain. J Comput Assist Tomogr, 13:20-26, 1989.

[15] P. A. Van den Elsen, E.-J. D. Pol, and M. A. Viergever. Medical image matching - A review with classification. IEEE Eng Med Biol Mag, pages 26-39, March 1993. 